This item was submitted to Loughborough's Research Repository by the author.

Items in Figshare are protected by copyright, with all rights reserved, unless otherwise indicated.

\title{
Disordered eating, compulsive exercise and sport participation in a UK adolescent sample
}

\section{PLEASE CITE THE PUBLISHED VERSION}

http://dx.doi.org/10.1002/erv.2441

\section{PUBLISHER}

(c) Wiley

\section{VERSION}

AM (Accepted Manuscript)

\section{PUBLISHER STATEMENT}

This work is made available according to the conditions of the Creative Commons Attribution-NonCommercialNoDerivatives 4.0 International (CC BY-NC-ND 4.0) licence. Full details of this licence are available at: https://creativecommons.org/licenses/by-nc-nd/4.0/

\section{LICENCE}

CC BY-NC-ND 4.0

\section{REPOSITORY RECORD}

Goodwin, Huw, Emma Haycraft, and Caroline Meyer. 2019. "Disordered Eating, Compulsive Exercise and Sport Participation in a UK Adolescent Sample". figshare. https://hdl.handle.net/2134/20279. 
Disordered eating, compulsive exercise and sport participation in a UK adolescent sample

\author{
Huw Goodwin Ph.D. ${ }^{1}$ \\ Emma Haycraft Ph.D. ${ }^{1 *}$ \\ Caroline Meyer Ph.D. ${ }^{2,3}$
}

${ }^{1}$ Loughborough University Centre for Research into Eating Disorders, School of Sport, Exercise and Health Sciences, Loughborough, Leicestershire, LE11 3TU

${ }^{2}$ Institute for Digital Healthcare, Warwick Manufacturing Group, University of Warwick, Coventry, CV4 7AL, UK

${ }^{3}$ Human Metabolism Research Unit, University Hospitals Coventry and Warwickshire NHS Trust, UK

* Address correspondence to: Dr Emma Haycraft, Loughborough University Centre for Research into Eating Disorders, School of Sport, Exercise and Health Sciences, Loughborough, Leicestershire, LE11 3TU.

Email: E.Haycraft@lboro.ac.uk; Tel. +44(0)1509 228160.

Running head: DISORDERED EATING AND ADOLESCENT SPORT 


\begin{abstract}
The sport literature has produced equivocal results as to whether sport participation is a protective or risk factor for disordered eating. One mechanism by which it could be a risk factor is the increased drive or compulsion to exercise. This study compared the levels of disordered eating and compulsive exercise between adolescent sport and non-sport participants. A sample of 417 male and female adolescents, aged 14-16 years old, was recruited from UK secondary schools. Participants completed questionnaire packs that included the Eating Disorder Inventory (EDI), a measure of exercise behaviour, and the Compulsive Exercise Test (CET). Non-sport participants reported significantly greater body dissatisfaction than sport participants, and this was true for boys and girls. Significant group differences were also reported for many of the CET scales, with sport participants generally reporting greater levels of compulsive exercise than non-sport participants. Implications of these results are discussed.
\end{abstract}

Keywords: Health, Psychology, Eating Disorders, Compulsive Exercise, Clinical Psychology, Body Image, 


\section{Disordered eating, compulsive exercise and sport participation in a UK adolescent}

\section{sample}

Some studies have shown increased levels of disordered eating among athletes compared to non-athlete groups (e.g., Hausenblas \& Carron, 1999; Sundgot-Borgen \& Torstveit, 2004). This effect is particularly well established among lean sport participants (Sundgot-Borgen, 1993, 1994a, 1994b). However, other research has reported no difference between athletes and non-athletes (e.g., Kirk, Singh, \& Getz, 2001). Therefore, it remains uncertain as to whether sport represents a risk or protective factor from the eating disorders, or whether it has any impact at all.

Where a positive link between sport and eating pathology has been found, it has been hypothesised that the association is a result of not only societal pressures, but also of sportspecific risk factors (Petrie \& Greenleaf, 2007), including pressure from coaches, greater focus on body shape and size, and revealing sport attire (e.g., Patel, Greydanus, Pratt, \& Phillips, 2003; Thompson \& Sherman, 2010). These sport-specific factors are likely to increase the athlete's focus on body weight and shape, as well as increase their desire to lose weight (Kerr, Berman, \& De Souza, 2006). Among female collegiate athletes, sport pressures regarding body weight and shape have recently been shown to predict body dissatisfaction (Anderson, Petrie, \& Neumann, 2011), which is regarded as one of the key risk factors in the development of eating disorders (Stice, Marti, \& Durant, 2011).

In addition to the sport-specific risks associated with negative body image and increased focus on weight described above, engaging in sport could also present as a risk factor for eating disorders through the increased volumes of exercise required for participation. Specifically, high volumes of exercise have been implicated in the aetiology of eating disorders (Davis, Kennedy, Ravelski, \& Dionne, 1994; Epling \& Pierce, 1996). However, it is important to note that researchers have found that it is not the exercise 
behaviour per se that is important in the development of eating disorders but rather the underpinning cognitions and motivations around exercise, such as exercising for weight and shape reasons, or cognitions around obligatory exercise (e.g., Cook \& Hausenblas, 2008; Seigel \& Hetta, 2001). Such findings suggest that it is important to measure cognitive and attitudinal aspects of exercise, such as compulsivity towards exercise (Meyer, Taranis, Goodwin, \& Haycraft, 2011), exercise addiction (Terry, Szabo, \& Griffiths, 2004), or exercise dependence (Hausenblas \& Symons Downs, 2002), when looking at the link between exercise and disordered eating. Specifically, within the eating disorder literature, the excessive and driven exercise behaviours are predominantly conceptualised as compulsive exercise (e.g., Adkins \& Keel, 2005; Meyer et al., 2011; Taranis \& Meyer, 2010), and so it would seem most appropriate to include compulsive exercise in studies examining disordered eating, exercise, and/or sport.

It is important to examine this relationship in adolescents, as this age group is critical in the development of the eating disorders (Striegel-Moore \& Bulik, 2007). However, much of the previous research has utilised adult samples, often from American University student or elite populations (see Hausenblas \& Carron, 1999). Studies that have examined sport and disordered eating among adolescents to date have also found equivocal results similar to the adult sample investigations. Several studies have found greater levels of disordered eating among athletic adolescents compared to non-athletic controls. For example, one study of Australian adolescent boys and girls found that sport involvement was associated with greater disordered eating (McCabe \& Ricciardelli, 2004). In addition, a previous investigation of female American high school athletes found that they were more likely than controls to possess behavioural and psychological correlates of eating disorders (Taub \& Blinde, 1992). Contrary to these results, a more recent investigation of Scandinavian high school students found that self-reported disordered eating was more prevalent among the control group than 
among the adolescent elite athletes (Martinsen, Bratland-Sanda, Eriksson, \& SundgotBorgen, 2010). The mechanisms that underpin these group differences are thought to be similar to those described above in the adult samples, although no study has compared sport participants versus non-sport participants on levels of compulsive exercise in an adolescent sample.

In summary, it is unclear whether sport participation presents as a risk or protective factor in relation to eating pathology. Previously identified associations between sport and eating disorder psychopathology (e.g., Sundgot-Borgen \& Torstveit, 2004) suggest that sport participation could be detrimental for some individuals. However, it is unknown whether this concern centres around the disordered eating, weight and shape attitudes that sport participants may be more vulnerable to, or whether sport participants have greater levels of compulsive exercise cognitions. This uncertainty in the literature needs to be examined in an adolescent population due to this age group's heightened risk for the eating disorders (Striegel-Moore \& Bulik, 2007). Therefore, the aim of this study is to compare adolescents involved in competitive sport with adolescents not involved in competitive sport on measures of disordered eating and compulsive exercise cognitions.

\section{Method}

\section{Participants}

A sample of 417 adolescents (boys $n=168$; girls $n=249$ ), recruited from secondary schools in the United Kingdom (UK), took part in an on-going research project into adolescent eating and exercise-related attitudes. The age of the sample ranged from 14 to 16 years old $(\mathrm{M}=14.80$ years; $\mathrm{SD}=.67)$, with there being homogeneity in terms of nationality (British $=97.3 \%$ ) and ethnicity ("White British" = 92.9\%). The socioeconomic status of the sample, as assessed by the location of the schools, found the recruitment areas to be of 
average to low levels of economic deprivation (Office for National Statistics, 2008).

Participants completed self-report measures of height and weight, which were converted into Body Mass Index (BMI) values. The mean BMI for boys was $21.12(\mathrm{SD}=3.06)$ and for girls it was $20.73(\mathrm{SD}=3.27)$. These values were then adjusted for age and gender into BMI $\mathrm{z}$ scores (Child Growth Foundation, 1996). The mean BMI z score for boys was 0.54 (SD = 1.08), whilst it was $0.15(\mathrm{SD}=1.11)$ for girls, suggesting generally healthy weights.

\section{Measures and Procedure}

Following ethical approval granted by the Institutional Review Board, participating schools received questionnaire packs. Questionnaire packs were completed at school during a class period by pupils within the study age range (i.e., 14-16 years old), supervised by a member of staff. The supervising member of staff was given standardised instructions to ensure consistent completion of questionnaires. The packs contained initial demographic information, including self-reported body height and weight, as well as self-reported exercise behaviour, using the Leisure Time Exercise Questionnaire (LTEQ; Godin \& Shephard, 1985). The LTEQ is a well-recognised method for assessing exercise behaviour, and has been validated against objective exercise measures (Eisenmann, Milburn, Jacobsen, \& Moore, 2002). The LTEQ has been previously used in UK samples of adolescent school pupils (e.g., Cumming, Standage, Gillison, \& Malina, 2008; Gillison, Standage, \& Skevington, 2006). The questionnaire packs then contained the following key measures:

Competitive sport participation. In accordance with the work of McCabe and Ricciardelli (2004), participants were asked whether or not they engaged in any competitive sport. For those who had answered yes to participating in sport, a conditional follow-up question asked which sport(s) they participated in and to what level of competition (e.g., club, county, regional, national, etc). To ensure consistency with previous research that has studied 
eating disorders in sport, the type of sport was then categorised into lean sports or non-lean sports (Sundgot-Borgen, 1993; 1994a; 1994b). The lean sports included sports that were aesthetic (e.g., gymnastics, diving, figure skating), weight-dependent (e.g., martial arts, wrestling, boxing), or endurance-based (e.g., running, cycling, swimming); since these sports all emphasise leanness. Non-lean sports comprised ball games (e.g., basketball, football, volleyball) or technical sports (e.g., golf, sailing), where leanness is not generally emphasised in relation to performance.

Compulsive exercise. Compulsive exercise was measured using the Compulsive Exercise Test (CET; Taranis, Touyz, \& Meyer, 2011). The CET consists of five subscales, constituting the key aspects of compulsive exercise. These include Avoidance and RuleDriven Behaviour (e.g., “I feel extremely guilty if I miss an exercise session”); Weight Control Exercise (e.g., “I exercise to burn calories and lose weight”); Mood Improvement Exercise (e.g., “I feel less anxious after I exercise”); Lack of Exercise Enjoyment (e.g., “I find exercise a chore”); and Exercise Rigidity (e.g., “I follow a set routine for my exercise”). Responses are scored using a 6-point Likert scale, ranging from '0 - never true' to '5 always true'. Mean item scores for each subscale are summed to form a total CET score. Higher scores equate to greater levels of compulsive exercise. The CET has been validated in an adolescent sample (Goodwin, Haycraft, Taranis, \& Meyer, 2011). The reliability of the CET scales for the current sample were .89 (Avoidance and Rule-Driven Behaviour), .82 (Weight Control Exercise), .84 (Mood Improvement Exercise), .76 (Lack of Exercise Enjoyment), .71 (Exercise Rigidity), and .89 (CET Total).

Disordered eating. Disordered eating was assessed using the Eating Disorder Inventory-2 (EDI; Garner, 1991). Only three of the subscales (Drive for Thinness, Bulimia, and Body Dissatisfaction) were used as these are regarded as the core features representing disordered eating attitudes (Garner, 1991). The use of these three scales as a measure of 
disordered eating has been reported in previous research (e.g., Haycraft \& Blissett, 2010). The EDI is a widely used measure of disordered eating and has displayed good psychometric properties for this age group (e.g., McCarthy, Simmons, Smith, Tomlinson, \& Hill, 2002). In the current sample, the Cronbach's alpha values were .89 (Drive for Thinness), .61 (Bulimia), and .92 (Body Dissatisfaction).

\section{Data Analysis}

An initial series of Kolmogorov-Smirnov tests revealed that the majority of continuous variables were not normally distributed, and therefore, non-parametric tests were used in all subsequent analyses. Preliminary analyses revealed significant differences between girls and boys on all study variables (Mann Whitney U test; $\mathrm{p}<.001$ ). Therefore, all analyses were conducted separately for boys and girls.

A second preliminary analysis was conducted among the sport participants only $(\mathrm{n}=$ 240) to compare the lean sport participants with the non-lean sport participants on the other study variables, also using a Mann Whitney U test. This was done to identify whether the type of sport had an impact on any potential sport versus non-sport differences. There were no significant differences between the lean and non-lean sport participants on BMI (z scores) or on any of the EDI subscales ( $p>.05$ ). Male lean sport participants reported significantly greater $(\mathrm{p}<.05)$ Avoidance and Rule-Driven Behaviour, Mood Improvement Exercise and CET Total than male non-lean sport participants, whilst among the girls, lean sport participants had significantly higher Exercise Rigidity scores than the non-lean sport participants $(\mathrm{p}<.05)$. Although there were these significant differences found on the CET subscales, the group sizes were particularly small, and therefore, no further comparisons were made using type of sport. 
The study's primary analysis was conducted using a non-parametric test of difference (Mann Whitney U test) comparing sport participants versus non-sport participants on the three EDI subscales, as well as the five CET subscales. Effect sizes $(r)$ were calculated for significant findings, with $0.10,0.30$, and 0.50 regarded as small, medium, and large effects, respectively (Cohen, 1992). Significance was set at $\mathrm{p}<.05$ for all tests.

\section{Results}

Descriptive Results

The descriptive statistics for the male and female sport and non-sport participants can be seen in Tables 1 and 2. Among boys, 69.6\% of participants were involved in competitive sport $(n=117)$. There were 26 different sports recorded as the principal sport participation, with the modal sports being football $(n=45)$ and rugby $(n=19)$. The majority of sport participants (60.1\%) competed at either club or county level. There were no significant differences on LTEQ or BMI z score data between the sport and non-sport participants (p $>.05$; see Table 1). The number of lean sport participants among the boys was 18, which was $15.8 \%$ of all male sport participants.

Among girls, 49.4\% ( $\mathrm{n}=123)$ were competitive sport participants. There were 20 sports recorded as the principal sport participated in. The modal sports reported for girls were hockey $(n=29)$, dance $(n=22)$, athletics $(n=14)$ and horse riding $(n=11)$. The majority of female sport participants (70.9\%) competed at school, club, or county level. Female sport participants reported significantly greater LTEQ scores than non-sport participants $(\mathrm{p}<.05$; see Table 1), with an effect size of $r=0.15$. There was no significant difference in BMI z scores between the sport and non-sport participants $(\mathrm{p}>.05)$. There were 71 female lean sport participants, which was $58.2 \%$ of all female sport participants. 
The mean scores for the study variables were largely comparable to other research. The BMI (not z scores) values were similar to another UK sample of school children (e.g., Cumming et al., 2008), although the current sample reported less activity on the LTEQ than the comparable UK sample (e.g., Cumming et al., 2008). The CET Total mean score for the girls in the current sample was larger than the boys' CET Total mean score, although the girls’ CET Total mean score was similar to another study investigating compulsive exercise in young women (Taranis et al., 2011), and represents mild levels of compulsive exercise cognitions. The EDI mean scores in the current sample were also slightly lower than found in other research that has studied adolescents (e.g., O’Dea \& Abraham, 1999), and represent low levels of disordered eating.

\section{Disordered Eating}

The means, standard deviations and tests of difference for the continuous study variables between sport and non-sport participants can be seen in Table 1. Among boys there was a significant difference between sport and non-sport participants on Body Dissatisfaction. Non-sport participants reported greater Body Dissatisfaction scores than sport participants, recording an effect size of $r=0.20$ (small to medium). There were no significant group differences for Drive for Thinness or Bulimia ( $p>.05)$. For girls, there was also a significant difference between the groups on Body Dissatisfaction, with Body Dissatisfaction found to be greater in non-sport participants than in sport participants. The effect size for this difference was $r=0.19$ (small to medium). There were no significant differences between groups on Drive for Thinness or Bulimia ( $\mathrm{p}>.05)$.

\section{Compulsive Exercise}


Table 2 shows the means, standard deviations and tests of difference between sport and non-sport participants for the CET subscales. Among the boys, Mann Whitney U tests found that there was a significant difference of small to medium effect sizes between sport participants and non-sport participants on the CET subscales of Avoidance and Rule-Driven Behaviour ( $r=0.36, \mathrm{p}<.001)$, Weight Control Exercise $(r=0.17, \mathrm{p}<.05)$, Mood Improvement Exercise $(r=0.27, p<.001)$, Lack of Exercise Enjoyment $(r=0.17, p<.05)$, Exercise Rigidity $(\mathrm{r}=0.33, \mathrm{p}<.001)$, and also for CET Total $(\mathrm{r}=0.31, \mathrm{p}<.001)$. For all CET subscales and CET Total, sport participants reported greater scores than non-sport participants, with the exception of Lack of Exercise Enjoyment, where the non-sport participants reported greater scores than the sport participants.

Among the girls, sport participants reported significantly greater scores than non-sport participants (small to medium effect sizes) on the CET subscales of Avoidance and RuleDriven Behaviour $(r=0.26, p<.001)$, Mood Improvement Exercise $(r=0.32, p<.001)$, Exercise Rigidity $(r=0.31, \mathrm{p}<.001)$, and also for CET Total $(\mathrm{r}=0.16, \mathrm{p}<.05)$. Non-sport participants reported significantly greater Lack of Exercise Enjoyment scores than sport participants $(\mathrm{r}=0.36, \mathrm{p}<.001)$. There was no significant difference between sport and nonsport participants on Weight Control Exercise ( $>$ >.05).

\section{Discussion}

The current study had the primary aim of investigating the difference between adolescent sport and non-sport participants on measures of disordered eating and compulsive exercise. Among both boys and girls, there was no significant difference between sport and non-sport participants on drive for thinness or bulimic attitudes. This is in line with previous studies that have found no difference between athletes and non-athletes on measures of disordered eating (e.g., Kirk et al., 2001), and thus suggesting that sport is neither a protective 
nor a risk factor for the core anorectic or bulimic cognitions associated with the development of eating disorders.

However, in the current sample, body dissatisfaction was significantly greater in those that were not involved in sport, compared to those who were regularly involved in sport. Sport could therefore represent a protective environment from the development of disordered eating in adolescence by offering a different body shape to the societal thin ideal to strive for, and/or reducing the individual's concern to strive for a thin body (Zucker, Womble, Williamson, \& Perrin, 1999). Sport could also offer greater satisfaction as bodies become more functional in nature and less about shape and appearance. This would support previous work with adolescent girls, which found that sport participants valued the functional nature of their bodies and were generally more satisfied with their bodies than were non-sport participants (Abbott \& Barber, 2011). A leaner body could be achieved by greater volumes of sport participation and this could be one way in which sport is associated with greater body satisfaction (although one may have a lean body prior to taking up sport). This leanness may not simply be about reduced weight, as there were no significant differences between the sport and non-sport participants in terms of BMI (z score) values. However, instead, muscular development may be also relevant, although this needs further investigation in its relation with body dissatisfaction, and the mechanism by which sport alters adolescents’ perceptions of their body shapes.

The current study added to the existing literature by investigating the difference between adolescent sport and non-sport participants on their levels of compulsive exercise. Importantly, those involved in sport reported significantly greater levels of compulsive exercise cognitions than did the adolescents who did not partake in competitive sport, and this was true for boys and girls. In particular, for boys, all aspects of compulsive exercise were significantly increased (with the exception of a lack of exercise enjoyment, which was 
decreased) among the sport participants. This suggests that male adolescent sport participants are more rigid with their exercise behaviours, generally feel low if they miss exercise, and also feel better when they are able to engage in exercise, than male adolescents who are not involved in sport. These cognitions may be healthy at low levels, leading to increased exercise behaviour, but if heightened to more clinical eating disorder levels could develop into psychologically and physically damaging behaviours (Meyer et al., 2011).

The compulsive exercise cognitions and group differences were similar for girls as well. One point of interest among the girls was the lack of a significant finding between the sport and non-sport participants on the CET scale of Weight Control Exercise. This suggests that regardless of whether or not girls are involved in sport, their motivation to exercise for controlling weight does not differ. This is important practically, as exercising for weight and shape, or 'food related' reasons have been associated with eating disorder psychopathology (Hubbard, Gray, \& Parker, 1998), and therefore, among girls, simply playing sport may have no effect on their weight control exercise, which in turn may have no effect on their level of risk for the eating disorders. This could be useful in informing eating disorder prevention work, although this finding requires replication in further research.

The difference between the groups on compulsive exercise cognitions highlights an association between competitive sport and compulsivity towards exercise, even at this relatively young age. These greater compulsive exercise cognitions among sport participants could represent the factor that puts competitive athletes at greater risk of developing eating disorders, as compulsive exercise has been implicated in the development of eating disorders (e.g., Davis et al., 1994). However, this association between sport, compulsive exercise, and disordered eating requires further research using a longitudinal design.

The findings from this study have some practical implications. Sport participation could represent a protective factor for the eating disorders by minimising levels of body 
dissatisfaction among adolescents. This is important as body dissatisfaction has been regarded as one of the biggest risk factors for the onset of eating disorders (Stice et al., 2011). The current findings suggest that there is something about playing sport that is associated with reduced feelings of body dissatisfaction, and this is true for boys and girls.

Consequently, it would appear that sport has this additional positive influence on adolescents, and as such should be further encouraged among this age group.

An additional practical implication is on the measurement of compulsive exercise. The lack of differences found for total exercise behaviour (LTEQ data) across several groups, even where there were significant compulsive exercise differences, demonstrates the importance of measuring compulsive exercise cognitively and affectively and not just through a behavioural measure of exercise volume. Researchers that have used behavioural indicators to define compulsive exercise (e.g., Brewerton, Stellefson, Hibbs, Hodges, \& Cochrane, 1995; Penas-Lledo, Leal, \& Waller, 2002) may miss out many individuals who have compulsive exercise cognitions but are not exercising in great volumes. Clinically, this could be problematic as defining compulsive exercisers based on exercise behaviour, such as hours per week of exercise, may mean that some compulsive exercisers who have strong cognitions around exercise may be overlooked and not receive the treatment they require.

It must be noted that this study did have some limitations. First, the exercise behaviour and body height and weight were all self-report data, in order to obtain information from a relatively large sample. This subjective measurement may have led to some of the non-significant findings, in particular the lack of a BMI difference between lean and non-lean sports. Future studies should look to replicate these findings with an objective measure of exercise behaviour and of height and weight. Second, the cross-sectional nature of the study precluded an ability to establish temporal precedence between the variables. As such, it was not possible to identify whether sport participation led to the decrease in body dissatisfaction 
and increase in compulsive exercise cognitions, or whether those with an existing satisfaction with their bodies and an existing compulsivity towards exercise chose to partake in sport, perhaps as a means of satisfying this compulsion. Finally, this study explored eating disordered attitudes and future research could extend this by exploring eating disordered behaviours.

Despite these limitations, this study does provide useful findings among a UK adolescent sample. Ultimately, the complex relationship between sport participation, disordered eating and compulsive exercise requires further research to elucidate whether competitive sport may be a risk or protective factor in the development of the eating disorders. 


\section{Acknowledgements}

The authors thank Laura Richards for her assistance with coding of the data. 


\section{References}

Abbott, B. D., \& Barber, B. L. (2011). Differences in functional and aesthetic body image between sedentary girls and girls involved in sports and physical activity: Does sport type make a difference? Psychology of Sport and Exercise, 12, 333-342.

Adkins, E. C., \& Keel, P. K. (2005). Does “excessive” or “compulsive” best describe exercise as a symptom of bulimia nervosa? International Journal of Eating Disorders, 38, 2429.

Anderson, C. M., Petrie, T., A., \& Neumann, C. S. (2011). Psychosocial correlates of bulimic symptoms among NCAA Division-I female collegiate gymnasts and swimmers/divers. Journal of Sport \& Exercise Psychology, 33, 483-505.

Brewerton, T. D., Stellefson, E. J., Hibbs, N., Hodges, E. L., \& Cochrane, C. E. (1995). Comparison of eating disorder patients with and without compulsive exercising. International Journal of Eating Disorders, 17, 413-416.

Child Growth Foundation. (1996). Cross Sectional Stature and Weight Reference Curves for the UK. London, United Kingdom: Child Growth Foundation.

Cohen, J. (1992). "A power primer". Psychological Bulletin, 112(1), 155-159.

Cook, B. J., \& Hausenblas, H. A. (2008). The role of exercise dependence for the relationship between exercise behaviour and eating pathology: Mediator or moderator? Journal of Health Psychology, 13, 495-502.

Cumming, S. P., Standage, M., Gillison, F., \& Malina, R. M. (2008). Sex differences in exercise behaviour during adolescence: Is biological maturation a confounding factor? Journal of Adolescent Health, 42, 480-485.

Davis, C., Kennedy, S. H., Ravelski, E., \& Dionne, M. (1994). The role of physical activity in the development and maintenance of eating disorders. Psychological Medicine, 24, 957-967. 
Eisenmann, J. C., Milburn, N., Jacobsen, L., \& Moore, S. (2002). Reliability and convergent validity of the Godin Leisure-Time Exercise Questionnaire in rural $5^{\text {th }}$ grade school children. Journal of Human Movement Studies, 43, 135-149.

Epling, W. F., \& Pierce, W. D. (1996). Activity anorexia: Theory, research, and treatment. New Jersey, USA: Lawrence Erlbaum.

Garner, D. M. (1991). EDI-2: Professional manual. Odessa, FL: Psychological Assessment Resources.

Gillison, F. B., Standage, M., \& Skevington, S. M. (2006). Relationships among adolescents' weight perceptions, exercise goals, exercise motivation, quality of life and leisure-time exercise behaviour: A self-determination theory approach. Health Education Research, $21,836$.

Godin, G., \& Shephard, R. J. (1985). A simple method to assess exercise behavior in the community. Canadian Journal of Applied Sport Sciences, 10, 141-146.

Goodwin, H., Haycraft, E., Taranis, L., \& Meyer, C. (2011). Psychometric evaluation of the Compulsive Exercise Test (CET) in an adolescent population: Links with eating psychopathology. European Eating Disorders Review, 19, 269-279.

Hausenblas, H. A., \& Carron, A. V. (1999). Eating disorder indices and athletes: An integration. Journal of Sport \& Exercise Psychology, 21, 230-258.

Hausenblas, H. A., \& Symons Downs, D. (2002). Exercise dependence: A systematic review. Psychology of Sport and Exercise, 3, 89-123.

Haycraft, E., \& Blissett, J. (2010). Eating disorder symptoms and parenting styles. Appetite, 54, 221-224.

Hubbard, S. T., Gray, J. J., \& Parker, S. (1998). Differences among women who exercise for ‘food related' and 'non-food related’ reasons. European Eating Disorders Review, 6, 255-265. 
Kerr, G., Berman, E., \& De Souza, M. J. (2006). Disordered eating in women’s gymnastics: Perspectives of athletes, coaches, parents, and judges. Journal of Applied Sport Psychology, 18, 28-43.

Kirk, G., Singh, K., \& Getz, H. (2001). Risk of eating disorders among female college athletes and non-athletes. Journal of College Counseling, 4, 122-132.

Martinsen, M., Bratland-Sanda, S., Eriksson, A. K., \& Sundgot-Borgen, J. (2010). Dieting to win or to be thin? A study of dieting and disordered eating among adolescent elite athletes and non-athlete controls. British Journal of Sports Medicine, 44, 70-76.

McCabe, M. P., \& Ricciardelli, L. A. (2004). A longitudinal study of pubertal timing and extreme body change behaviours among adolescent boys and girls. Adolescence, 39, 145-167.

McCarthy, D. M., Simmons, J. R., Smith, G. T., Tomlinson, K., \& Hill, K. A. (2002). Reliability, stability, and factorial invariance of the Eating Disorder Inventory II and the Bulimia Test-Revised in adolescence. Assessment, 9, 382-389.

Meyer, C., Taranis, L., Goodwin, H., \& Haycraft, E. (2011). Compulsive exercise and eating disorders. European Eating Disorders Review, 19, 174-189.

O’Dea, J. A., \& Abraham, S. (1999). Onset of disordered eating attitudes and behaviours in early adolescence: Interplay of pubertal status, gender, weight, and age. Adolescence, 34, 671-679.

Office for National Statistics (2008). Neighbourhood Statistics. Office for National Statistics.

Retrieved February 15, 2009 from http://neighbourhood.statistics.gov.uk/

Pallant, J. (2007). SPSS survival manual ( ${ }^{\text {rd }}$ edition). New York: McGraw-Hill.

Patel, D., Greydanus, D. E., Pratt, H. D., \& Phillips, E. L. (2003). Eating disorders in adolescent athletes. Journal of Adolescent Research, 18, 280-296. 
Peñas-Lledó, E., Leal, F. J. V., \& Waller, G. (2002). Excessive exercise in anorexia nervosa and bulimia nervosa: Relation to eating characteristics and general psychopathology. International Journal of Eating Disorders, 31, 370-375.

Petrie, T. A., \& Greenleaf, C. (2007). Eating disorders in sport: From theory to research to intervention. In G. Tenenbaum \& R. Eklund (Eds.), Handbook of sport psychology (pp. 352-378). Hoboken, NJ: John Wiley \& Sons.

Seigel, K., \& Hetta, J. (2001). Exercise and eating disorder symptoms among young females. Eating \& Weight Disorders, 6, 32-39.

Stice, E., Marti, C. N., \& Durant, S. (2011). Risk factors for onset of eating disorders: Evidence of multiple risk pathways from an 8-year prospective study. Behaviour Research and Therapy, 49, 622-627.

Striegel-Moore, R. H., \& Bulik, C. M. (2007). Risk factors for eating disorders. American Psychologist, 62, 181.

Sundgot-Borgen, J., \& Torstveit, M. K. (2004). Prevalence of eating disorders in elite athletes is higher than in the general population. Clinical Journal of Sport Medicine, 14, 25.

Sundgot-Borgen, J. (1993). Prevalence of eating disorders in elite female athletes.

International Journal of Sport Nutrition, 3, 29-29.

Sundgot-Borgen, J. (1994a). Eating disorders in female athletes. Sports Medicine, 17, 176188.

Sundgot-Borgen, J. (1994b). Risk and trigger factors for the development of eating disorders in female elite athletes. Medicine \& Science in Sports \& Exercise, 26, 414.

Taranis, L., \& Meyer, C. (2010). Associations between specific components of compulsive exercise and eating-disordered cognitions and behaviors among young women. International Journal of Eating Disorders, 44, 452-458. 
Taranis, L., Touyz, S., \& Meyer, C. (2011). Disordered eating and exercise: Development and preliminary validation of the Compulsive Exercise Test (CET).European Eating Disorders Review, 19, 256-268.

Taub, D. E., \& Blinde, E. M. (1992). Eating disorders among adolescent female athletes: Influence of athletic participation and sport team membership. Adolescence, 27, 833848.

Terry, A., Szabo, A., \& Griffiths, M. (2004). The exercise addiction inventory: A new brief screening tool. Addiction Research \& Theory, 12, 489-499.

Thompson, R. A., \& Sherman, R. T. (2010). Eating disorders in sport. New York: Routledge.

Zucker, N. L., Womble, L. G., Williamson, D., A., \& Perrin, L. A. (1999). Protective factors for eating disorders in female college athletes. Eating Disorders, 7, 207-218. 


\section{TABLE 1}

Descriptive statistics and tests of difference between sport and non-sport participants on Body Mass Index, Exercise Behaviour, and Eating Disorder Inventory subscales

\begin{tabular}{|c|c|c|c|c|}
\hline & $\begin{array}{c}\text { Sport } \\
\text { Participants }\end{array}$ & $\begin{array}{c}\text { Non-Sport } \\
\text { Participants Mean }\end{array}$ & $\begin{array}{l}\text { Test of difference } \\
\text { (z) }\end{array}$ & ES \\
\hline Variable & Mean (SD) & (SD) & & \\
\hline Boys & $n=117$ & $n=51$ & & \\
\hline BMI z scores & $0.57(1.08)$ & $0.46(1.08)$ & 0.72 & Ns \\
\hline Total Exercise Behaviour (LTEQ) $^{a}$ & $68.58(34.72)$ & $61.98(49.36)$ & 1.42 & Ns \\
\hline EDI Drive for Thinness & $1.46(2.66)$ & $1.80(3.27)$ & 0.09 & Ns \\
\hline EDI Bulimia & $1.10(1.74)$ & $1.49(2.84)$ & 0.05 & Ns \\
\hline EDI Body Dissatisfaction & $3.61(4.47)$ & $6.41(6.43)$ & $2.61 * *$ & 0.20 \\
\hline Girls & $n=123$ & $n=126$ & & \\
\hline BMI z scores & $0.11(1.09)$ & $0.20(1.14)$ & 0.73 & Ns \\
\hline Total Exercise Behaviour $^{a}$ & $58.76(41.13)$ & $48.92(39.28)$ & $2.32 *$ & 0.15 \\
\hline EDI Drive for Thinness & $4.77(5.71)$ & $6.05(6.31)$ & 1.47 & Ns \\
\hline EDI Bulimia & $1.95(2.86)$ & $2.31(2.82)$ & 1.14 & Ns \\
\hline EDI Body Dissatisfaction & $9.03(7.53)$ & $12.21(8.56)$ & $2.94 * *$ & 0.19 \\
\hline
\end{tabular}

Note. BMI = Body Mass Index; EDI = Eating Disorder Inventory; LTEQ = Leisure Time Exercise Questionnaire; ES = Effect Size (r); ns = Non-significant.

${ }^{\mathrm{a}}$ Measured in Metabolic Equivalents (METS).

${ }^{*} \mathrm{p}<.05 .{ }^{* *} \mathrm{p}<.01 .{ }^{* * *} \mathrm{p}<.001$ 


\section{TABLE 2}

Descriptive statistics and tests of difference between sport and non-sport participants on the Compulsive Exercise Test subscales.

\begin{tabular}{|c|c|c|c|c|}
\hline & $\begin{array}{c}\text { Sport Participants } \\
\text { Mean (SD) }\end{array}$ & $\begin{array}{l}\text { Non-Sport } \\
\text { Participants }\end{array}$ & $\begin{array}{c}\text { Test of } \\
\text { difference }\end{array}$ & ES \\
\hline Variable & & Mean (SD) & (z) & \\
\hline Boys & $n=117$ & $n=51$ & & \\
\hline Avoidance and Rule-Driven & $1.47(1.11)$ & $0.71(0.75)$ & $4.61 * * *$ & 0.36 \\
\hline \multicolumn{5}{|l|}{ Behaviour } \\
\hline Weight Control Exercise & $1.85(1.00)$ & $1.47(0.97)$ & $2.24 *$ & 0.17 \\
\hline Mood Improvement Exercise & $2.47(1.13)$ & $1.72(1.28)$ & $3.48 * * *$ & 0.27 \\
\hline Lack of Exercise Enjoyment & $0.87(0.81)$ & $1.25(1.05)$ & $2.19 *$ & 0.17 \\
\hline Exercise Rigidity & $2.22(1.21)$ & $1.35(1.17)$ & $4.23 * * *$ & 0.33 \\
\hline CET Total & $8.87(3.43)$ & $6.50(3.28)$ & $4.01 * * *$ & 0.31 \\
\hline Girls & $n=123$ & $n=126$ & & \\
\hline Avoidance and Rule-Driven & $1.43(0.96)$ & $0.99(0.91)$ & $4.06 * * *$ & 0.26 \\
\hline \multicolumn{5}{|l|}{ Behaviour } \\
\hline Weight Control Exercise & $2.41(1.23)$ & $2.60(1.33)$ & 1.16 & Ns \\
\hline Mood Improvement Exercise & $2.88(1.05)$ & $2.14(1.13)$ & $5.04 * * *$ & 0.32 \\
\hline Lack of Exercise Enjoyment & $1.05(0.93)$ & $1.78(1.10)$ & $5.62 * * *$ & 0.36 \\
\hline Exercise Rigidity & $2.53(1.15)$ & $1.79(1.13)$ & $4.93 * * *$ & 0.31 \\
\hline CET Total & $10.30(3.17)$ & $9.30(3.52)$ & $2.50^{*}$ & 0.16 \\
\hline
\end{tabular}

Note . CET = Compulsive Exercise Test; ES = Effect Size (r); ns = Non-significant. ${ }^{*} \mathrm{p}<.05 .{ }^{* *} \mathrm{p}<.01 .{ }^{* * *} \mathrm{p}<.001$. 